\title{
Evaluation of a Dedicated Dual Phased-Array Surface Coil Using a Black-Blood FSE Sequence for High Resolution MRI of the Carotid Vessel Wall
}

\author{
Mohamed Ouhlous, MD, ${ }^{1}$ Franck Lethimonnier, $\mathrm{PhD},{ }^{2}$ Diederik W.J. Dippel, MD, $\mathrm{PhD},{ }^{3}$ \\ Marc R.H.M. van Sambeek, MD, PhD, ${ }^{4}$ Lambertus C.J. van Heerebeek, RT, ${ }^{1}$ \\ Peter M.T. Pattynama, MD, PhD, ${ }^{1}$ and Aad van der Lugt, MD, $\mathrm{PhD}^{1 *}$
}

\begin{abstract}
Purpose: To investigate the ability of magnetic resonance imaging (MRI) to visualize the carotid vessel wall using a phased-array coil and a black-blood (BB) fast spin-echo (FSE) sequence.
\end{abstract}

\begin{abstract}
Materials and Methods: The phased-array coil was compared with a three-inch coil. Images from volunteers were evaluated for artifacts, wall layers, and wall signal intensity. Signal intensity and homogeneity of atherosclerosis were assessed. Lumen diameter and vessel area were measured.

Results: Comparison between the phased-array coil and the three-inch coil showed a $100 \%$ increase in signal-tonoise ratio. BB-FSE imaging resulted in good delineation between blood and vessel wall. Most volunteers had a twolayered vessel wall with a hyperintense inner layer. MRI showed both homogeneous hyperintense and heterogeneous plaques, which consisted of a main hyperintense part with hypointense spots and/or intermediate regions. MRI lumen and area measurements were performed easily.
\end{abstract}

Conclusion: High resolution MRI of carotid atherosclerosis is feasible with a phased-array coil and a BB-FSE sequence.

Key Words: magnetic resonance imaging; black-blood fast spin-echo; phased-array coil; carotid artery; atherosclerosis J. Magn. Reson. Imaging 2002;15:344-351.

(c) 2002 Wiley-Liss, Inc.

CEREBROVASCULAR ACCIDENTS are related to atherosclerosis of the carotid artery. In particular, the severity of the stenosis is a predictor of clinical symptoms

\footnotetext{
${ }^{1}$ Department of Radiology, University Hospital Rotterdam, Rotterdam, The Netherlands.

${ }^{2}$ GE Medical Systems, Buc, France.

${ }^{3}$ Department of Neurology, University Hospital Rotterdam, Rotterdam, The Netherlands.

${ }^{4}$ Department of Surgery, University Hospital Rotterdam, Rotterdam, The Netherlands.

Contract grant sponsor: Van Leersumfonds, KNAW, The Netherlands. *Address reprint requests to: A.vdL., Department of Radiology, University Hospital Rotterdam, Dr. Molewaterplein 40, 3015 GD Rotterdam, The Netherlands. E-mail: vanderlugt@rond.azr.nl

Received July 3, 2001; Accepted November 6, 2001.
}

and is used as a parameter in the therapeutical decision as to which patient will benefit from carotid endarterectomy $(1,2)$. In addition to the severity of the atherosclerotic stenosis, plaque morphology is considered an independent predictor of stroke (3-5).

Due to its inherent superior contrast resolution, magnetic resonance imaging (MRI) has the potential to provide a surplus of information on the composition of the atherosclerotic plaque. Previous in vitro validation studies have shown that differentiation of calcifications, fibrocellular tissue, lipids, and intraplaque hemorrhage (thrombus) is possible (6-13).

In vivo imaging of the atherosclerotic plaque in the carotid artery requires surface coils and dedicated pulse sequences to provide high-resolution images with good delineation of the lumen and the vessel wall, without disturbance from flow artifacts (14-17). Flow artifacts may be addressed by a black-blood (BB) double inversion recovery (IR) technique, which effectively removes signal from flowing blood (18-19). In this study, the performance of a new dual phased-array coil was evaluated. A BB fast spin-echo (BB-FSE) pulse sequence was tested in volunteers and patients, and the ability of MRI to provide morphologic and quantitative parameters of atherosclerotic disease in the carotid arteries was assessed.

\section{MATERIALS AND METHODS}

\section{Dual Phased-Array Surface Coil}

A receive-only dual phased-array coil (custom made) was designed and constructed specifically to create a limited penetration depth with high signal-to-noise ratio (SNR). The coil assembly consisted of two partly overlapping square coils of $50 \times 50 \mathrm{~mm}$. This classical phased-array pair was matched to present $50 \mathrm{Ohms}$ to the scanner interface when coils were loaded with the patient. During the duration of the transmit pulse, the coils were actively detuned by means of positive-intrinsicnegative (PIN) diodes, which prevent large circulating currents in the coils during the transmit pulse. Additional safety was provided by a pair of crossed diodes 
Table 1

Imaging Protocol Used in the Present Study

\begin{tabular}{lcccc}
\hline \multicolumn{1}{c}{ Parameters } & 2D TOF GRE & PDw BB-FSE & Partial T2w BB-FSE & T2w BB-FSE \\
\hline TR $(\mathrm{msec})$ & 24 & 2 heart beats & 2 heart beats & 2 heart beats \\
TE $(\mathrm{msec})$ & 4.8 & 14 & 43 & 85 \\
BW $(\mathrm{kHz})$ & 20 & 20 & 20 & 20 \\
FOV $(\mathrm{mm})$ & 200 & 60 & 60 & 60 \\
Slice thickness $(\mathrm{mm})$ & 3 & 3 & 3 & 3 \\
Matrix & $256 \times 160$ & $256 \times 256$ & $256 \times 256$ & $256 \times 256$ \\
NEX & 1 & 2 & 2 & 2 \\
ETL & - & Cardiac & 8 & 16 \\
Gating & - & $234 \times 234$ & $234 \times 234$ & Cardiac \\
Slices & 25 & 8 & 8 & 8 \\
Resolution $(\mu \mathrm{m})$ & - & & $234 \times 234$ \\
Scan time $(\mathrm{min})$ & 2 & & 8 \\
\hline
\end{tabular}

that became active in decoupling the coils in the unlikely event of failure of the PIN diodes. The total required capacitance of the coil was distributed over the coil to minimize frequency shift due to the loading of the coil by the patient. The coil was constructed from flexible silver plate, supported by an epoxy board. The assembly was coated with a soft polymer foam to minimize patient discomfort. The coil was used on both sides of the neck and was held in place with a soft collar.

\section{Pulse Sequences}

All images were acquired on a 1.5-T MR scanner (General Electric, Milwaukee, WI; Signa $\mathrm{CV} / i$, with an amplitude of $40 \mathrm{mTm}^{-1}$ and a slew rate of $150 \mathrm{Tm}^{-1} \mathrm{~s}^{-1}$ ). The imaging protocol consisted of the following sequences (Table 1). First, a T2* fast gradient-recalled echo sequence (FGRE; TR $65 \mathrm{msec}$, TE $2.3 \mathrm{msec}$, field of view [FOV] $26 \mathrm{~cm}$, matrix $256 \times 128$, number of excitations [NEX] 2) was used in axial, sagittal, and coronal planes as localizer. The sagittal image, which showed the cervical vertebral bodies, was used to plan a two-dimensional time-of-flight (TOF) GRE sequence (Fig. 1). Twentyfive slices with a slice thickness of $3 \mathrm{~mm}$ were set between cervical vertebrae C2-C6 using the phasedarray coil as receiver. A marker was placed in the center of the dual phased-array coil to assess the position of the coil in relation to the carotid bifurcation and the atherosclerotic plaque (Fig. 1). If necessary, the coil was adjusted and the two-dimensional TOF sequence was repeated.

The caudal coordinates of the bifurcation on the twodimensional TOF GRE images were used to plan consecutive axial 3-mm proton density weighted (PDw) BBFSE slices with the carotid artery in the center of the image. The first slice was planned just below the bifurcation; the next slices cranially, without interslice spacing. In the patient studies, T2-weighted (T2w) BB-FSE images were acquired at the levels with atherosclerotic disease seen on the PDw images.

The BB sequence was described first by Edelman et al in 1991 for gradient echo sequences and subsequently by Simonetti et al in 1996 for FSE sequences $(18,19)$. The cardiac-gated BB-FSE sequence uses a non-selective and a slice-selective IR preparation before the FSE acquisition starts to reduce the blood signal and related artifacts. The non-selective IR pulse inverts the magnetization of the entire body, including blood. The second IR pulse, which is slice-selective, is immediately applied and re-inverts the imaging slice. The magnetization from outside the imaging slice (most notably that of the blood) is inverted and magnetization within the slice is essentially unchanged. A standard FSE sequence is played after a delay time in which the blood that was originally in the imaging slice that was re-inverted flows out of the imaging slice, and the inverted blood becomes
Figure 1. Left panel: Sagital T2*FGRE localizer that was used to plan the two-dimensional-TOF sequence between vertebrae C2-C6 (lines). Right panel: Two-dimensional TOF image of the carotid bifurcation, which was used to plan the axial BB-FSE images. A marker (arrow) shows the position of the coil in relation to the bifurcation.




Table 2

Increase (\%) in SNR Between 3-Inch Surface Coil and Dual Phased-Array Coil Measurements in a Phantom at the Center and Off-Center at Different Depths

\begin{tabular}{cccccccc}
\hline $\begin{array}{c}\text { Depth } \\
(\mathrm{cm})\end{array}$ & $\begin{array}{c}\text { Right } \\
3 \mathrm{~cm}\end{array}$ & $2 \mathrm{~cm}$ & $1 \mathrm{~cm}$ & $\begin{array}{c}\text { Center } \\
0\end{array}$ & $1 \mathrm{~cm}$ & $2 \mathrm{~cm}$ & $\begin{array}{c}\text { Left } \\
3 \mathrm{~cm}\end{array}$ \\
\hline 1 & 234 & 173 & 141 & 127 & 135 & 154 & 208 \\
2 & 290 & 155 & 132 & 119 & 127 & 154 & 204 \\
3 & 144 & 127 & 112 & 107 & 91 & 139 & 178 \\
4 & 117 & 103 & 93 & 93 & 80 & 91 & 137 \\
5 & 91 & 85 & 81 & 68 & 68 & 77 \\
6 & 73 & 75 & 69 & & & 77 \\
\hline
\end{tabular}

nulled and flows into the imaging slice. The double-IR preparation and FSE acquisition is typically repeated using $2 \times$ RR cardiac gating until all the shots of the FSE acquisition are acquired. By applying the double-IR preparation just after the cardiac trigger, the slice-selective re-inversion pulse is placed in the "end diastolic' part of the cardiac cycle. Because the inversion time is usually close to $650 \mathrm{msec}$, the FSE acquisition is also played out during diastole (19).

\section{Studies \\ Dual Phased-Array Coil Vs. Standard Three-Inch Single-Loop Coil}

Phantom studies were performed with dual phasedarray coil and a standard three-inch single-loop coil (General Electric, Milwaukee, WI) using a GRE pulse sequence $(\mathrm{TR}=100 \mathrm{msec}, \mathrm{TE}=7 \mathrm{msec}$, flip angle $=30$, FOV $15 \times 15 \mathrm{~cm}$, matrix $256 \times 256,1 \mathrm{NEX}$, and $3-\mathrm{mm}$ slice thickness). SNR for both coils was assessed by mapping a phantom filled with copper sulfate. Using a GE workstation (ADW 3.0 software package), circular regions of interest (ROIs) were positioned on the phantom images and the mean signal was assessed at the center of the phantom and off-center $(-3,-2,-1,1,2$, and $3 \mathrm{~cm})$ at different depths $(1,2,3,4,5$, and $6 \mathrm{~cm})$. An estimation of noise was derived from the standard deviation $\left(\mathrm{SD}_{\mathrm{air}}\right)$ of a small circular $\mathrm{ROI}$ placed on the air surrounding the phantom. SNR was calculated using the formula: $\left(0.655 \times\right.$ signal) $/ \mathrm{SD}_{\text {air }}(20,21)$. An estimation of SNR increase was calculated by: $\left(\mathrm{SNR}_{\mathrm{d}}-\mathrm{SNR}_{\mathrm{i}} /\right.$ $\left.\mathrm{SNR}_{\mathrm{i}}\right) \times 100(\mathrm{~d}=$ dual phased-array coil, $\mathrm{i}=$ three-inch surface coil).

Studies in volunteers $(N=5)$ and patients $(N=3)$ were performed with both coils using partial T2w BBFSE (TE $=45 \mathrm{msec}$ ). The study was approved by the Ethical Committee. All volunteer and patient studies were performed after written informed consent was obtained. Two observers independently assessed the image quality of both coils using a score of 1 to 3 ( $1=\mathrm{bad}$, $2=$ moderate, and 3 = good). Interobserver agreement in the quality assessment was calculated using Kappa statistics.

\section{Volunteers}

Fifteen volunteers (mean age 26.8 years, range 19-59 years, three females) were imaged with the phasedarray coil using $\mathrm{PDw}(\mathrm{TE}=14 \mathrm{msec})$ and $\mathrm{T} 2 \mathrm{w}(\mathrm{TE}=85$ msec) BB-FSE sequences. Two images of the PDw BB-
FSE series were analyzed per study; the first image at the bifurcation, and the second image $6 \mathrm{~mm}$ higher in the internal carotid artery (ICA). Two observers assessed the delineation between lumen and vessel wall and evaluated the presence of artifacts, the number of layers that could be distinguished within the carotid vessel wall and the intensity of the inner vessel wall layer (hyperintense, intermediate, and hypointense compared to surrounding muscles).

\section{Patients}

Ten patients (mean age 66.9 years, range $49-80$ years, all males) with symptomatic atherosclerotic disease of the carotid artery were imaged with the phased-array coil using PDw (TE = $14 \mathrm{msec})$ and $\mathrm{T} 2 \mathrm{w}(\mathrm{TE}=85 \mathrm{msec})$ BB-FSE sequences. The ability to visualize the presence of atherosclerotic lesion was assessed. On each PDw image, homogeneity and signal intensities of the atherosclerotic plaque (hyperintense, intermediate, and/or hypointense) were assessed and used to characterize the plaques. Alterations in signal intensities were also assessed on the corresponding T2w images.

Lumen area (LA) and vessel area (VA) at the most stenotic site (smallest lumen) in the PDw BB-FSE images was measured on a GE workstation (ADW 3.0 software package). The contours of the lumen and vessel area were manually traced. The plaque area (PA) was calculated by subtracting LA from VA. The degree of stenosis was assessed by the ratio of PA/VA. Lumen area was also measured in the most cranial image in a plaque-free ICA. Lumen diameter (LD) was calculated from the lumen area measurements using the formula: $\mathrm{LD}=\mathrm{V}(4 \cdot \mathrm{LA} / \pi)$. The lumen stenosis was assessed by the formula: $\mathrm{LD}_{\mathrm{c}}-\mathrm{LD}_{\mathrm{s}} / \mathrm{LD}_{\mathrm{c}}\left(\mathrm{LD}_{\mathrm{s}}=\right.$ lumen diameter at most stenotic lumen and $\mathrm{LD}_{\mathrm{c}}=$ lumen diameter cranially in the ICA). MRI data were compared with angiographic images in which the severity of stenosis was measured using the North American Symptomatic Cartoid Encarterectomy Trial (NASCET) criteria (1).

\section{RESULTS \\ Dual Phased-Array Coil Vs. Standard Three-Inch Single-Loop Coil}

In the phantom study, the dual phased-array coil had a better SNR than the three-inch surface coil at all measured depths at the center and off-center (Table 2). At the depth of the carotid artery $(2.5$ to $3.5 \mathrm{~cm})$, the dual 
Figure 2. Two partial T2w BB-FSE images $(3 \mathrm{~mm})$ of carotid bifurcation in a healthy volunteer obtained with the three-inch surface coil (left panel) and the phased-array coil (right panel). Images reveal a hyperintense inner layer surrounded by a hypointense outer layer. (ICA = internal carotid artery; ECA = external carotid artery; JV = jugular vein).

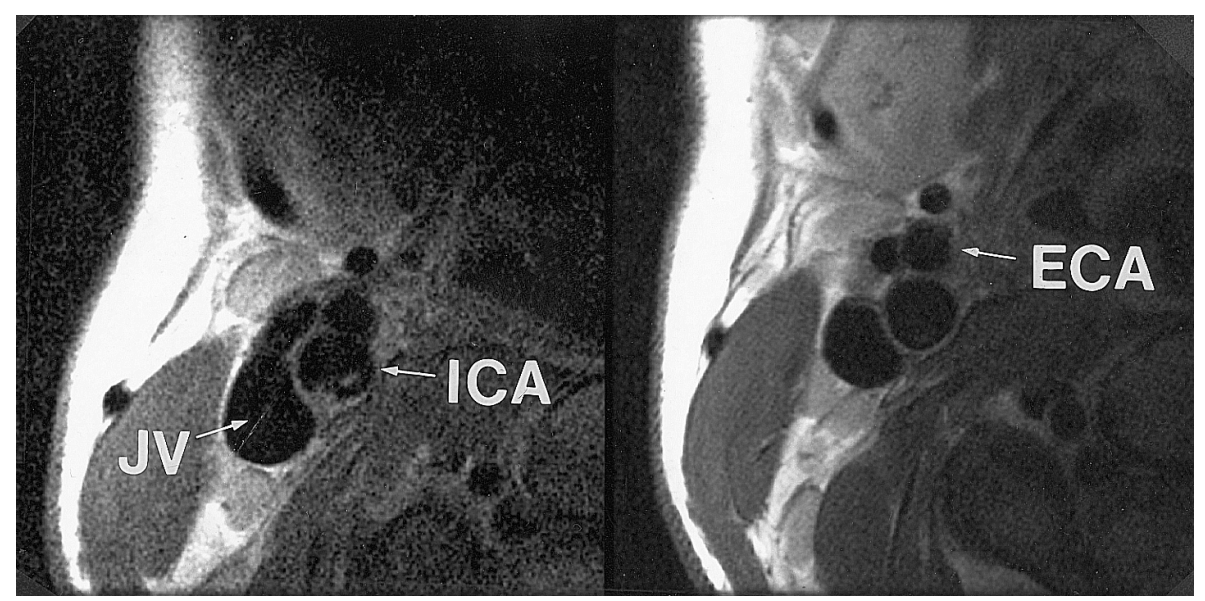

phased-array coil showed an increase in SNR of more than $100 \%$.

In the in vivo studies, the phased-array coil was judged better than the standard three-inch coil with regard to image quality (Fig. 2). In one study, the phased-array coil was judged worse due to motion artifacts. There was a fair agreement between the two observers on the image quality of both coils (Table 3). The observers agreed totally on the comparison of both coils.

\section{Volunteers}

The phased-array coil was easy to hold in position using a soft collar. With the imaging protocol, it was possible to cover up a distance of approximately $2.5 \mathrm{~cm}$ of the carotid artery. In 23\% of the images obtained in volunteers, an intraluminal artifact (a high signal) was experienced in the carotid bulbus at the posterior site of the internal carotid artery (Fig. 3). Retrospective analysis revealed that this was related to the application of peripheral gating instead of electrocardiogram (ECG) gating. On both the PDw and T2w images, the observers could distinguish two layers in the wall of a normal carotid artery in $85 \%$ of the volunteers, with a high $(80 \%)$ or an intermediate signal intensity in the inner layer. The inner layer of the vessel wall was surrounded by a thin hypointense outer layer (Figs. 2-4). Four images from two patients revealed a hypointense arterial

Table 3

Interobserver Variability in the Assessment of Image Quality

\begin{tabular}{llccc}
\hline & Quality & $\begin{array}{c}\text { Observer } \\
1\end{array}$ & $\begin{array}{c}\text { Observer } \\
2\end{array}$ & Kappa \\
& & & & \\
$\begin{array}{l}\text { Dual phased- } \\
\text { array coil }\end{array}$ & Good & 6 & 4 & $\mathrm{~K}=0.56$ \\
& Moderate & 1 & 3 & \\
& Bad & 1 & 1 & \\
Single-loop & & & & $\mathrm{K}=0.48$ \\
surface coil & Good & 4 & 1 & \\
& Moderate & 2 & 4 & \\
Phased-array vs. & Bad & 2 & 3 & \\
surface coil & Better & 7 & 7 & $\mathrm{~K}=1.0$ \\
& Worse & 1 & 1 & \\
\hline & & & &
\end{tabular}

layer with no distinction between an inner and outer layer.

The comparison between the two observers with regard to flow artifacts, number of layers seen within the carotid vessel wall, and inner layer intensity, is summarized in Table 4. The agreement between the observers was very good for the two-layered appearance of the vessel wall and for the intensity of the inner layer.

\section{Patients}

Most of the patients had an elongated plaque, which started in the common carotid artery with the smallest lumen usually at the origin of the ICA. Subsequently, the size of the plaque decreased and the vessel lumen returned to normal caliber cranially. Although the first image was planned below the bifurcation, in one patient it turned out to be in the ICA.

Seventy-six PDw images (six to eight per patient) were acquired. In two patients, only six slices were imaged due to a slow heart rate, which increased the scan time. Two images were degraded by motion artifacts; 18 images revealed a normal arterial wall (as seen in the

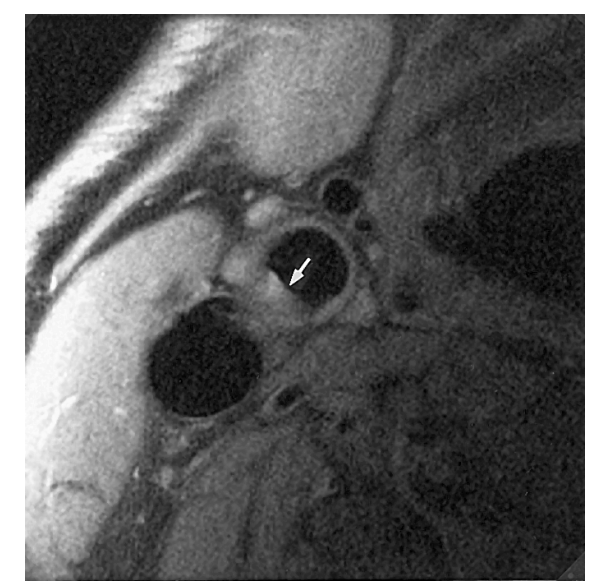

Figure 3. Peripheral cardiac-gated PDw BB-FSE image of a volunteer with a plaque-mimicking flow artifact (arrow) in the common carotid artery. The inner layer of the common carotid artery was hyperintense and surrounded by a hypointense outer layer. 


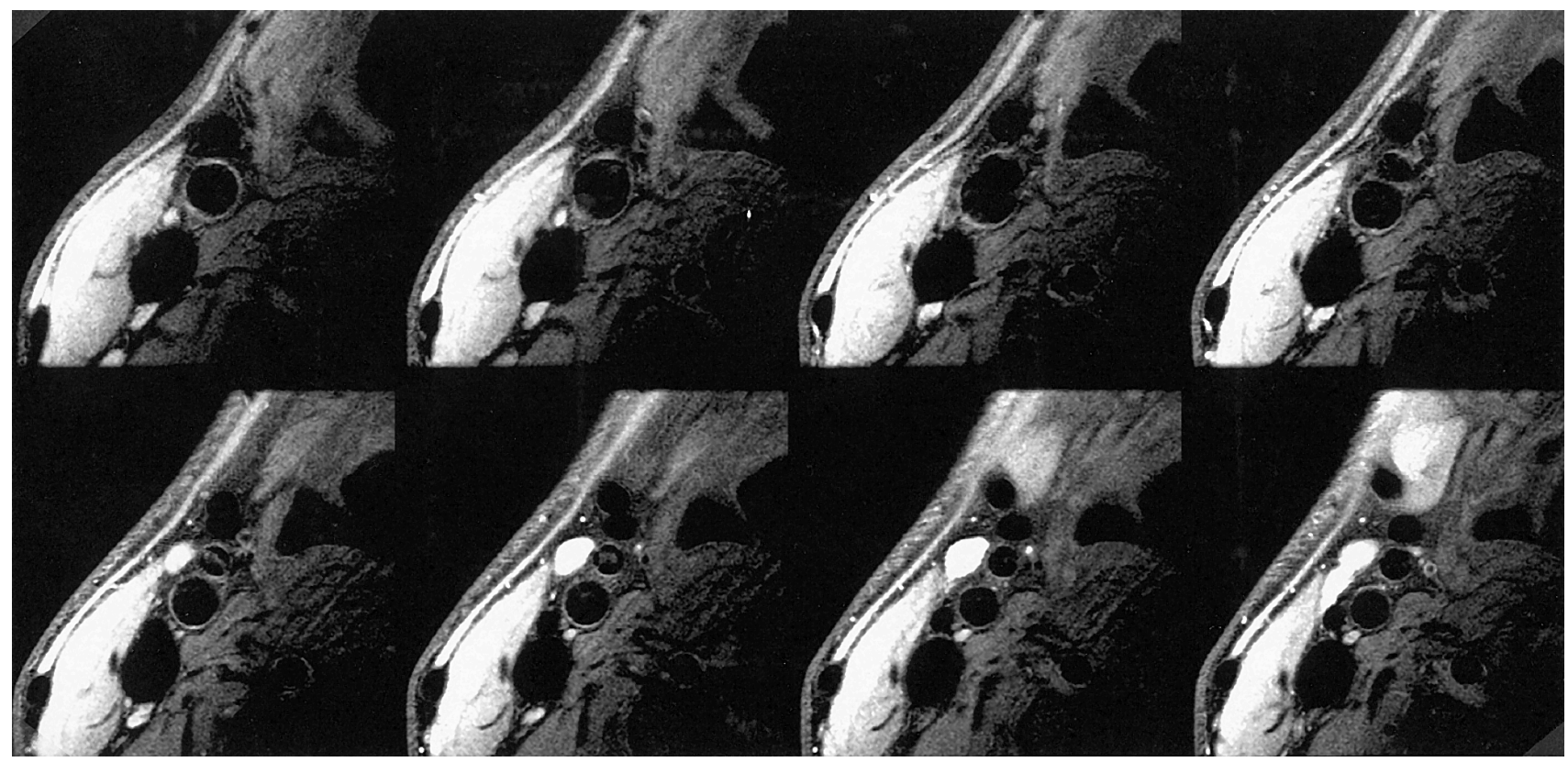

Figure 4. Eight consecutive PDw BB-FSE images of a volunteer.

volunteers). Atherosclerotic lesions were revealed in 56 PDw images (four to eight per patient). The original two-layered structure, which was visualized in most non-diseased vessel walls, could not be detected in the diseased vessel segments: the signal intensity of the inner layer (media) corresponded with the signal intensity of the atherosclerotic plaque. In the PDw images, the main part of the atherosclerotic plaque was visualized as a hyperintense region within the vessel wall boundaries (Table 5). A homogeneous hyperintense plaque was seen in 24 images and heterogeneous plaque was seen in 32 images (Fig. 5). Hypointense spots and intermediate regions were seen in 14 and 29 of these 32 images, respectively (Fig. 6). All hypointense spots and some intermediate regions were located inside the plaque, and surrounded by hyperintense regions. Some intermediate regions were located at the luminal site of the plaque (Figs. 5 and 6).

At the location of the atherosclerotic lesion (56 PDw images), 50 T2w images were acquired: 11 images were degraded by motion artifacts or low signal intensity. The remaining $39 \mathrm{~T} 2 \mathrm{w}$ images (zero to seven per pa-

Table 4

Interobserver Variability in the Assessment of Artefacts, Number of Layers and Intensity of the Inner Layer of the Carotid Vessel Wall

\begin{tabular}{lccc}
\hline & Observer 1 & Observer 2 & Kappa \\
\hline Artefacts & & & \\
Yes & 7 & 8 & $\mathrm{~K}=0.89$ \\
$\quad$ No & 23 & 5 & \\
Number of layers & & & $\mathrm{K}=0.87$ \\
1 & 4 & 25 & \\
2 & 26 & 22 & $\mathrm{~K}=0.91$ \\
Inner layer & & 6 & \\
$\quad$ Hyperintense & 23 & 2 & \\
$\quad$ Intermediate & 5 & & \\
$\quad$ Hypointense & 2 & &
\end{tabular}

tient) were compared with the corresponding PDw images. As expected, the T2w images had a lower SNR than the PDw images. The hyperintense and hypointense signal intensities seen on the PDw image did not change on the T2w images. Eight of the 17 intermediate regions at the luminal site retained their intermediate signal intensity, while nine regions became hypointense. For the intraplaque intermediate regions, eight of 13 regions kept their intermediate signal intensity and five regions became hypointense (Table 5).

The area measurements of the MR images are given in Table 6. At the site of the most severe stenosis, all patients had more than $85 \%$ area stenosis. The plaque area had a wide range from 25.0 to $84.1 \mathrm{~mm}^{2}$. On MRI, the luminal diameter stenosis (measured according to the NASCET criteria) was 31\%-81\%. On angiography, the severity of stenosis ranged from 59\%-83\%. The differences between the lumen diameter stenosis assessed with MRI and angiography ranged from 1\%-37\% (median $=6 \%$ ).

\section{DISCUSSION}

Surface coils are necessary to perform high resolution MRI of the atherosclerotic plaque in the carotid artery in

Table 5

Classification of the Morphology of the Plaque on the PDw/T2w Images

\begin{tabular}{lcc}
\hline & $\begin{array}{c}\text { PDw } \\
(N=56)\end{array}$ & $\begin{array}{c}\text { PDw/T2w } \\
(N=39)\end{array}$ \\
\hline Hyperintense & 56 & 39 \\
Homogeneous & 24 & 14 \\
Hypointense & 14 & 10 \\
Intermediate & 29 & 23 \\
$\quad$ Luminal site & 19 & 17 (hypointense 9; intermediate 8) \\
$\quad$ Intraplaque & 17 & 13 (hypointense 5; intermediate 8) \\
\hline a7 slices contained intermediate signal intensity at both sites.
\end{tabular}


Figure 5. PDw BB-FSE images showing an atherosclerotic plaque in the ICA. The plaque appeared to be eccentric and the signal was homogeneous hyperintense. Vessel lumen was surrounded by a region of intermediate signal intensity (arrow).



vivo. The current study compared the performance of a new dual phased-array coil with a standard single-loop surface coil. With the new coil, the distal common carotid artery, the bifurcation, and the proximal ICA could be covered with an increased SNR (factor of two) at the depth of the carotid artery. Hayes et al previously demonstrated that a phased-array coil is the preferred design for carotid imaging, as it offers a high SNR, good patient acceptance, ease of placement, and an adaptability to wide variation in patient anatomy. Their phased-array coil showed the same increase in SNR compared with a three-inch coil as the present study (22).
The BB FSE sequence was able to image the carotid artery with surrounding anatomy in a reasonable scan time. With this sequence, a clear distinction between lumen and vessel wall was obtained when ECG-gating was applied to suppress the signal of flowing blood. The first studies were performed with peripheral gating, which resulted in plaque-mimicking artifacts. With peripheral gating, the IR pulses are applied after the systolic pulse wave in the carotid artery, while with ECG gating, the IR pulses are applied just before the systolic pulse wave. This results in data acquisition before or during the consecutive systole with peripheral gating, while with ECG gating, the data are acquired during
Figure 6. PDw (upper panels) and corresponding $\mathrm{T} 2 \mathrm{w}$ (lower panels) images showing the common carotid artery (CCA) (left panels) and the ICA (right panels) with atherosclerotic disease. The mean part of the plaque revealed a hyperintense signal on PDw and T2w images. The plaque in the CCA revealed a region with intermediate signal intensity on PDw images, which became hypointense on T2w images (arrow). The plaque in the ICA revealed hypointense spots on PDw images and regions with intermediate signal intensity at the luminal site and inside the plaque. The intraplaque regions became hypointense on $\mathrm{T} 2 \mathrm{w}$ images, while the regions at the luminal site remained an intermediate signal intensity.

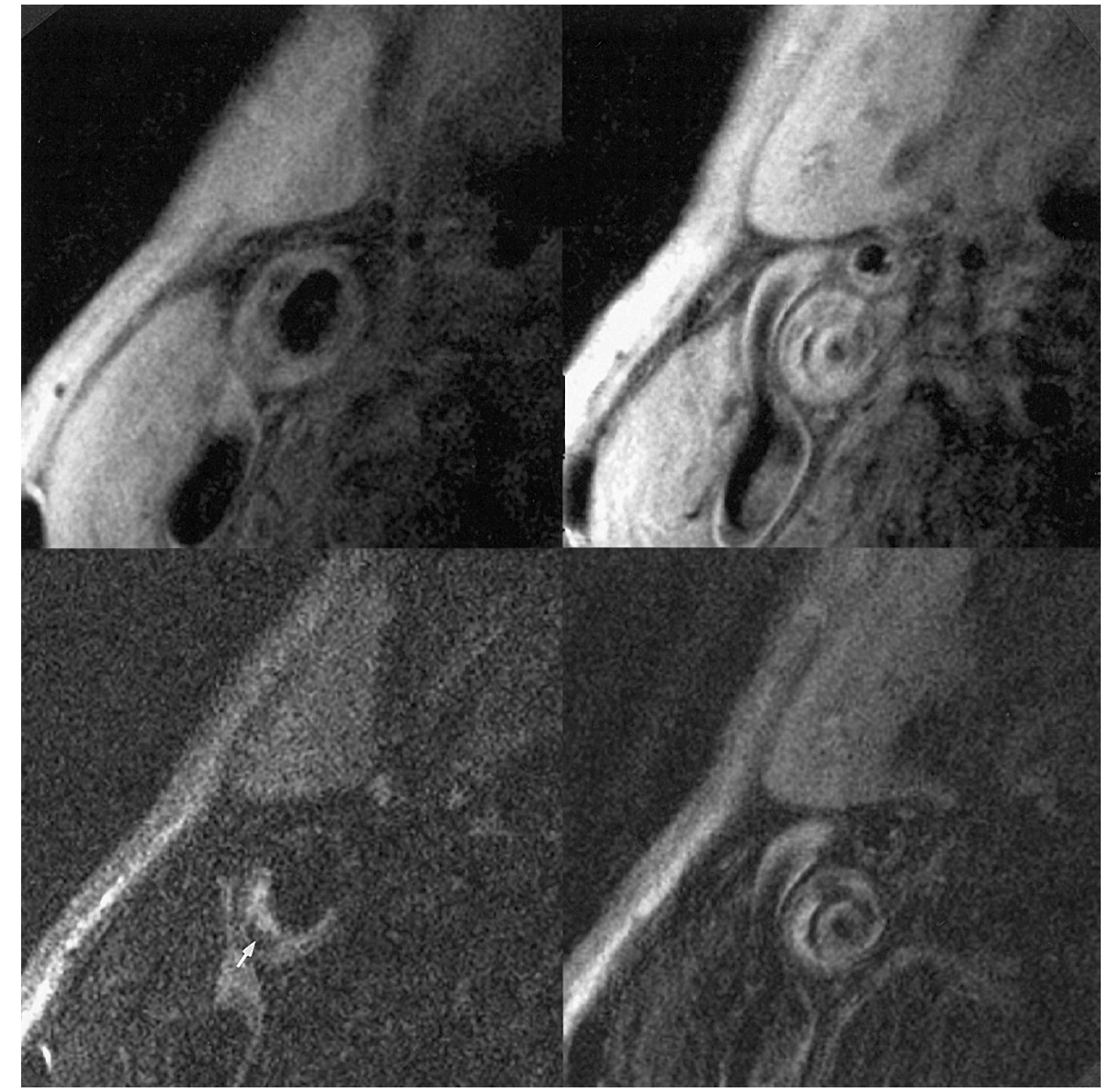


Table 6

Area and Diameter Measurements Assessed in MR Images of the Carotid Artery and Corresponding Angiographic Measurements

\begin{tabular}{|c|c|c|c|c|c|c|c|}
\hline \multirow[b]{3}{*}{ Patient } & \multicolumn{4}{|c|}{ MRI } & \multicolumn{3}{|c|}{ Angiography } \\
\hline & \multicolumn{4}{|c|}{ Most stenotic site } & \multicolumn{3}{|c|}{ Normal site } \\
\hline & $\begin{array}{l}\text { Lumen } \\
\text { area } \\
\left(\mathrm{mm}^{2}\right)\end{array}$ & $\begin{array}{l}\text { Vessel } \\
\text { area } \\
\left(\mathrm{mm}^{2}\right)\end{array}$ & $\begin{array}{l}\text { Plaque } \\
\text { area } \\
\left(\mathrm{mm}^{2}\right)\end{array}$ & $\begin{array}{c}\text { Area } \\
\text { stenosis } \\
(\%)\end{array}$ & $\begin{array}{l}\text { Lumen } \\
\text { area } \\
\left(\mathrm{mm}^{2}\right)\end{array}$ & $\begin{array}{c}\text { Lumen } \\
\text { diameter } \\
\text { stenosis (\%) }\end{array}$ & $\begin{array}{c}\text { Lumen } \\
\text { diameter } \\
\text { stenosis (\%) }\end{array}$ \\
\hline 1 & 4.1 & 29.1 & 25.0 & 86 & 8.7 & 31 & $\mathrm{np}$ \\
\hline 2 & 2.5 & 31.1 & 28.6 & 92 & 22.8 & 67 & 59 \\
\hline 3 & 1.1 & 48.5 & 47.4 & 98 & 9.3 & 66 & 68 \\
\hline 4 & 5.2 & 81.8 & 76.6 & 94 & 17.0 & 45 & 82 \\
\hline 5 & 2.3 & 37.4 & 35.1 & 94 & 16.8 & 63 & 74 \\
\hline 6 & 1.3 & 63.6 & 62.3 & 98 & 23.8 & 77 & 83 \\
\hline 7 & 0.6 & 70.7 & 70.1 & 99 & 15.6 & 81 & 75 \\
\hline 8 & 4.1 & 88.2 & 84.1 & 95 & 22.8 & 58 & $\mathrm{np}$ \\
\hline 9 & 1.1 & 67.3 & 66.2 & 98 & 24.1 & 79 & $\mathrm{np}$ \\
\hline 10 & 1.9 & 33.8 & 31.9 & 94 & 16.4 & 66 & 65 \\
\hline
\end{tabular}

$\mathrm{np}$, not performed.

diastole. In the first case, re-inverted blood in the imaging plane has not flown out of this plane fully.

Another BB imaging technique is the application of presaturation bands placed superior and inferior to the area of study. In this technique, a trigger delay is applied that results in data acquisition between peak systole and early diastole $(14,23)$. The advantage of this technique is that it allows multislice acquisition, which is not applicable in BB-FSE sequences. However, a disadvantage is the decrease in reliability of presaturation in the case of slow flowing blood (23).

Most of our studies in volunteers showed a two-layered construction of the carotid vessel wall with a hyperintense inner layer and a hypointense outer layer in the PDw and T2w images. This was also reported in other in vivo studies of the carotid vessel wall $(14,15)$. In vitro studies have shown that the hyperintense inner layer corresponds to intima and media, and the hypointense outer layer corresponds to the adventitia $(7,9)$. In some volunteers, the observers could not distinguish the two layers of the carotid vessel wall, but the carotid wall still has a higher signal intensity than the surrounding anatomy.

The combination of the BB-FSE sequence and the dual phased-array coil was able to visualize the atherosclerotic plaque and to distinguish it from lumen. The different signal intensities seen on PDw and T2w images suggest the presence of different plaque components, as described previously (14-16).

Hyperintense signal intensities on PDw that do not lose their signal on T2w images may indicate the presence of fibrous tissue, which has the highest T2 of the plaque components $(6,15)$. Inside the plaque, small hypointense spots were visualized on PDw images; this was probably caused by calcifications. Previous in vivo and in vitro studies demonstrated that calcifications were seen as regions with hypointense signal intensity or signal voids on all analyzed sequences (6-16). However, it is not certain whether small amounts of calcifications will be visualized as a hypointense region. With a slice thickness of $3 \mathrm{~mm}$, small calcifications may be visualized as intermediate signal intensities due to partial volume effects. Some of the intraplaque intermedi- ate signal intensities on PDw may be caused by such calcifications.

The intraplaque regions with an intermediate signal intensity on the PDw could also be caused by lipid, thrombus, or intraplaque hemorrhage. Lipid pools are located inside the plaque and studies have reported a lower T2 for lipid than for fibrous tissue. Shinnar et al reported that lipid and thrombus could not be distinguished from fibrous tissue on PDw images in contrast to $\mathrm{T} 2 \mathrm{w}$ images (TE $>30 \mathrm{msec}$ ); their study was performed on a 9.4-T scanner with a spin-echo sequence (6). Our study with a FSE sequence and an echo-train length of 16 may cause blurring of the images and may give stronger influence of tissues with shorter T2 (lipid, thrombus) on the signal intensity in PDw images, resulting in an intermediate signal.

The intraplaque structures, visualized with an intermediate signal on the PDw images, were shown as hypointense or intermediate intense regions on the T2w images. Toussaint et al reported that intraplaque hemorrhage had a lower T2 than lipid (15), which may explain the difference in the $\mathrm{T} 2 \mathrm{w}$ images.

The intermediate region at the luminal site of the plaque on the PDw images may be caused by thrombus. An intermediate region at the luminal site of the vessel wall on PDw images was also found to be a thrombus by Toussaint et al (15). The thrombus has a short T2 if it is fresh. During later organization of the atherosclerotic plaque, the T2 increases due to degradation of iron in deoxyhemoglobin. This may explain the changes in signal intensity of the intermediate regions on T2w images: the regions that lose their intermediate signal on the $\mathrm{T} 2 \mathrm{w}$ sequence may indicate the presence of a fresh thrombus, and regions that maintain an intermediate signal intensity may correspond to an old organized thrombus.

Besides the assessment of different components of the plaque, MRI was able to quantify the degree of stenosis. MRI lumen and area measurements could be performed easily. Angiography can accurately determine the luminal stenosis, but due to enlargement of the vessel area, the lumen can maintain its normal size with increase in atherosclerotic plaque (24). Moreover, 
angiography measures lumen diameter in one direction (anterior-posterior direction) and does not take into account the shape of the vessel lumen. This may explain the difference in diameter degree of stenosis measured using MRI and angiography. High resolution MRI offers the possibility to assess plaque area, which may be a better parameter to clinically monitor lesion progression and regression $(25,26)$.

In conclusion, high resolution MR images of the carotid artery can be obtained with a phased-array coil. BB FSE sequence results in a good delineation between lumen and vessel wall. Atherosclerotic plaque components could be visualized with MRI. High resolution MRI of the carotid artery opens a new window for the qualification, quantification, and monitoring of atherosclerotic lesions in vivo.

\section{ACKNOWLEDGMENTS}

The authors thank Herman Flick (Flick Engineering Solutions Inc., Utrecht, The Netherlands) for providing the dual phased-array coil.

\section{REFERENCES}

1. North American Symptomatic Carotid Endarterectomy Trial Collaborators. Benefit of carotid endarterectomy in patients with symptomatic moderate or severe stenosis. N Engl J Med 1998;339:14151425.

2. European Carotid Surgery Trialists' Collaborative. Randomised trial endarterectomy for recently symptomatic carotid stenosis: final results of the MRC European Carotid Surgery Trial (ECST). Lancet 1998;351:1379-1387.

3. Bassiouny HS, Sakagushi Y, Mikucki S, et al. Juxtalumenal location of plaque necrosis and neoformation in symptomatic carotid stenosis. J Vasc Surg 1997;26:585-594.

4. Park AE, McCarthy WJ, Pearce WH, et al. Carotid plaque morphology correlates with presenting symptomatology. J Vasc Surg 1998; 27:872-879.

5. Golledge J, Greenhalgh RM, Davies AH. The symptomatic carotid plaque. Stroke 2000;31:774-781.

6. Shinnar M, Fallon JT, Wehrli S, et al. The diagnostic accuracy of ex vivo MRI for human atherosclerotic plaque characterization. Arterioscler Thromb Vasc Biol 1999;19:2756-2761.

7. Martin AJ, Gotlieb AI, Henkelman RM. High resolution MR of human arteries. J Magn Reson Imaging 1995;5:93-100.

8. Gold GE, Pauly JM, Glover GH, et al. Characterization of atherosclerosis with a 1.5-T imaging system. J Magn Reson Imaging 1993; 3:399-407.
9. Toussaint JF, Southern JF, Fuster V, et al. T2-weighted contrast for NMR characterization of human atherosclerosis. Arterioscler Thromb Vasc Biol 1995; 15:1533-1542.

10. Yuan C, Petty C, O'Brien KD, et al. In vitro and in situ magnetic resonance imaging signal features of atherosclerotic plaque-associated lipids. Arterioscler Thromb Vasc Biol 1997;17:1496-1503.

11. Halliburton SS, Paschal CB. Atherosclerotic plaque components in human aortas by ex vivo imaging using fast spin-echo magnetic resonance imaging and spiral computed tomography. Invest Radiol 1996;31:724-728.

12. Yuan C, Tsuruda JS, Beach KN, et al. Techniques for high resolution MR imaging of atherosclerotic plaque. J Magn Reson Imaging 1994;4:43-49.

13. Winn WB, Schmiedl UP, Reichenbach DD, et al. Detection and characterization of atherosclerotic fibrous caps with T2-weighted MR. AJNR Am J Neuroradiol 1998;19:129-134.

14. Yuan C, Murakami JW, Hayes CE, et al. Phased array MRI of the carotid artery bifurcation: preliminary results in healthy volunteers and a patient with atherosclerosis disease. J Magn Reson Imaging 1995;5:561-565.

15. Toussaint JF, LaMuraglia GR, Southern JF, et al. Magnetic resonance images lipid, fibrous, calcified, hemorrhagic, and thrombotic components of human atherosclerosis in vivo. Circulation 1996;94: 932-938.

16. Von Ingersleben G, Schmiedl UP, Hatsukami TS, et al. Characterization of atherosclerotic plaques at the carotid bifurcation: correlation of high resolution MR imaging with histologic analysispreliminary study. Radiographics 1997;17:1417-1423.

17. Luk-Pat GT, Gold GE, Olcott EW, et al. High resolution threedimensional in vivo imaging of atherosclerotic plaque. Magn Reson Med 1999;42:762-771.

18. Edelman RR, Chien D, Kim D. Fast selective black blood MR imaging. Radiology 1991;181:421-426.

19. Simonetti OP, Finn JP, White RD, et al. "Black blood" T2-weighted inversion recovery MR imaging of the heart. Radiology 1996;199: 49-57.

20. Kaufman L, Kramer D, Crooks LE, et al. Measuring signal-to-noise ratios in MR imaging. Radiology 1989;173:265-267.

21. Firbank MJ, Harrison RM, Williams ED, et al. Quality assurance for MRI: practical experience. Br J Radiol 2000;73:376-383.

22. Hayes CE, Mathis CM, Yuan C. Surface coil phased arrays for high-resolution imaging of the carotid arteries. J Magn Reson Imaging 1996;1:109-112.

23. Steinman DA, Rutt BK. On the nature and reduction of plaquemimicking flow artifacts in black blood MRI of the carotid bifurcation. Magn Reson Med 1998;39:635-641.

24. Glagov S, Weisenberg E, Zarins CK, et al. Compensatory enlargement of human atherosclerotic coronary arteries. N Engl J Med 1987;316:1371-1375.

25. Yuan C, Beach KW, Smith LH, et al. Measurement of atherosclerotic carotid plaque size in vivo using high resolution magnetic resonance imaging. Circulation 1998;24:2666-2671.

26. Kang X, Polissar NL, Han C, et al. Analysis of the measurement precision of arterial lumen and wall areas using high-resolution MRI. Magn Reson Med 2000;44:968-972. 